\title{
Rare decay results and prospects with LHCb
}

\author{
Matteo Palutan* \\ (on behalf of the LHCb Collaboration) \\ INFN Laboratori nazionali di Frascati, Italy \\ E-mail: matteo.palutandlnf.infn.it
}

The search for the decays $B_{s}^{0} \rightarrow \mu^{+} \mu^{-}$and $B_{d}^{0} \rightarrow \mu^{+} \mu^{-}$performed with the LHCb experiment at the Large Hadron Collider at CERN is described. The study is based on $37 \mathrm{pb}^{-1}$ of $p p$ collisions collected at $\sqrt{s}=7 \mathrm{TeV}$. The observed numbers of events are consistent with the background expectations. The resulting upper limits on the branching fractions are $\operatorname{BR}\left(B_{s}^{0} \rightarrow \mu^{+} \mu^{-}\right)<5.6 \times$ $10^{-8}$ and $\operatorname{BR}\left(B_{d}^{0} \rightarrow \mu^{+} \mu^{-}\right)<1.5 \times 10^{-8}$ at $95 \%$ confidence level, and are competitive with the best world limits, from the Tevatron experiments. The LHCb prospects for the study of the decay $B_{d}^{0} \rightarrow K^{* 0} \mu^{+} \mu^{-}$in the near future are also briefly discussed.

The 13th International Conference on B-Physics at Hadron Machines - Beauty2011, April 04-08, 2011

Amsterdam, The Netherlands

${ }^{*}$ Speaker. 


\section{Search for the rare decays $B_{s}^{0} \rightarrow \mu^{+} \mu^{-}$and $B_{d}^{0} \rightarrow \mu^{+} \mu^{-}$}

The main goal of the LHCb experiment at the CERN Large Hadron Collider is to perform precision tests of the Standard Model (SM) in the flavour sector, namely the decays of the $b$ hadrons, with enough sensitivity to disentangle possible new physics effects. One of the most promising results in the initial phase of the LHCb physics program is certainly the search for the very rare dimuon decays of the $B_{d}^{0}$ and $B_{s}^{0}$ mesons, which are expected with high interest by the high energy physics community. Within the SM these decays, which are in addition helicity suppressed, can only happen through loop diagrams (see figure 1). They can have very different branching fractions (BR) in new physics models, especially in those with an extended Higgs sector. In particular, in supersymmetric models large enhancements are possible [1].
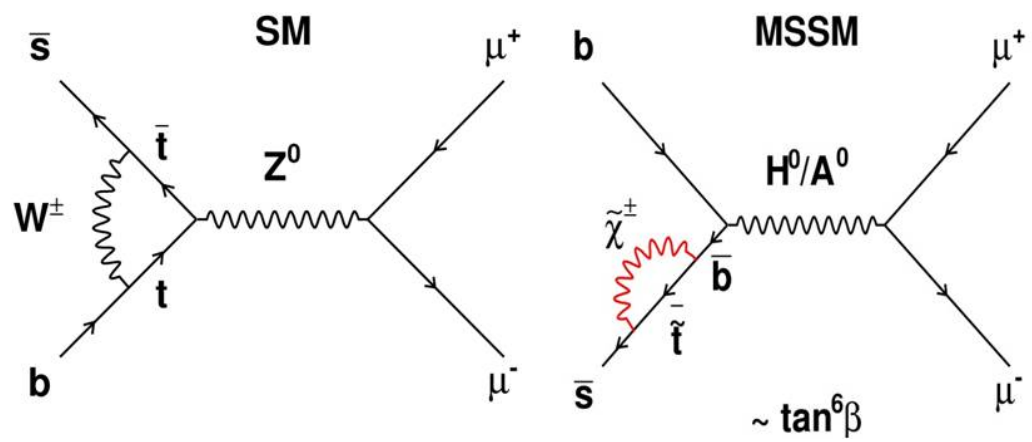

Figure 1: Feynman diagrams of contribution to the branching fraction within the SM (left) and within the MSSM with R-parity conservation (right).

The SM predictions have a relative precision of less than 10\% [2]:

$$
\begin{aligned}
& \mathrm{BR}\left(B_{s}^{0} \rightarrow \mu^{+} \mu^{-}\right)_{\mathrm{SM}}=(3.2 \pm 0.2) \times 10^{-9} \\
& \mathrm{BR}\left(B_{d}^{0} \rightarrow \mu^{+} \mu^{-}\right)_{\mathrm{SM}}=(1.0 \pm 0.1) \times 10^{-10} .
\end{aligned}
$$

The search for $B_{q}^{0} \rightarrow \mu^{+} \mu^{-}$has been performed both at $e^{+} e^{-}$colliders and at the Tevatron. The highest sensitivity so far has been achieved at the Tevatron due to the very large $b \bar{b}$ cross section at hadron colliders. Using $3.7 \mathrm{fb}^{-1}$ of data, the CDF collaboration pushed the limits down to $\mathrm{BR}\left(B_{s}^{0} \rightarrow \mu^{+} \mu^{-}\right)<43 \times 10^{-9}$ and $\mathrm{BR}\left(B_{d}^{0} \rightarrow \mu^{+} \mu^{-}\right)<7.6 \times 10^{-9}$, at $95 \%$ C.L. [3]

The LHCb experiment [4] is very well suited for such searches due to its excellent invariant mass resolution, vertex resolution, muon identification and trigger acceptance. The forward geometry of LHCb allows indeed the first level trigger to collect events containing one or two muons with very low transverse momenta $\left(p_{\mathrm{T}}\right)$ : more than $90 \%$ of the data were collected with a $p_{\mathrm{T}}$ threshold of $1.4 \mathrm{GeV} / c$ for single muon triggers and $p_{\mathrm{T}}\left(\mu_{1}\right)>0.48 \mathrm{GeV} / c$ and $p_{\mathrm{T}}\left(\mu_{2}\right)>0.56 \mathrm{GeV} / c$ for dimuon triggers. Moreover, among the LHC experiments, $\mathrm{LHCb}$ has the unique trigger capability of providing large samples of hadronic $B_{q}^{0} \rightarrow h^{+} h^{-}$decays. These events are used as control samples in order to reduce the dependence of the results on the detector simulation. The measurement described here uses an integrated luminosity of about $37 \mathrm{pb}^{-1}$ collected with the LHCb detector between July and October 2010 at $\sqrt{s}=7 \mathrm{TeV}$. Assuming the SM branching fraction, and using the $b \bar{b}$ cross-section measured within the LHCb acceptance of $75 \pm 14 \mu b$ [5], about 0.7 (0.08) $B_{s}^{0} \rightarrow \mu^{+} \mu^{-}\left(B_{d}^{0} \rightarrow \mu^{+} \mu^{-}\right)$are expected to be reconstructed. 


\subsection{Analysis strategy}

The first step of the analysis consists of a loose selection, which significantly reduces the size of the data set by rejecting most of the background. The second step consists of the study of three normalization channels: $B^{+} \rightarrow J / \psi\left(\mu^{+} \mu^{-}\right) K^{+}, B_{s}^{0} \rightarrow J / \psi\left(\mu^{+} \mu^{-}\right) \phi\left(K^{+} K^{-}\right)$and $B_{d}^{0} \rightarrow K^{+} \pi^{-}$. Using these normalization channels the $B \rightarrow \mu \mu$ branching fractions can be calculated from the observed signal events $N_{\text {sig }}$ as:

$$
\mathrm{BR}_{\text {sig }}=\mathrm{BR}_{\text {norm }} \times \frac{\varepsilon_{\text {norm }}^{\mathrm{REC}} \varepsilon_{\text {norm }}^{\mathrm{SEL} \mid \mathrm{REC}} \varepsilon_{\text {norm }}^{\mathrm{TRIG} \mid \mathrm{SEL}}}{\varepsilon_{\mathrm{sig}}^{\mathrm{REC}} \varepsilon_{\mathrm{sig}}^{\mathrm{SEL} \mid \mathrm{REC}} \varepsilon_{\mathrm{sig}}^{\mathrm{TRIG} \mid \mathrm{SEL}}} \times \frac{f_{\text {norm }}}{f_{B_{q}}} \times \frac{N_{\text {sig }}}{N_{\text {norm }}}=\alpha \times N_{\text {sig }},
$$

where the subscript denotes the signal or normalization channel, and the fragmentation fraction $f_{x}$ gives the probability that a $b$-quark hadronizes into the specified $b$-hadron. The reconstruction efficiency $\left(\varepsilon^{\mathrm{REC}}\right)$ includes the acceptance and particle identification, while $\varepsilon^{\mathrm{SEL} \mid \mathrm{REC}}$ denotes the selection efficiency on reconstucted events. The trigger efficiency on selected events is denoted by $\varepsilon^{\mathrm{TRIG} \mid \mathrm{SEL}}$. The usage of the normalization channels ensures that the knowledge of the absolute luminosity and total cross-section is not needed for the determination of the branching fraction, and that many systematic uncertainties cancel in the ratio of the efficiencies. The selection procedure for the normalization channels is specifically designed to be as close as possible to the signal selection. The ratios of reconstruction and selection efficiencies are estimated from MC, while the ratio of trigger efficiencies on selected events is determined on data. Results from the three normalization channels are in agreement within each other, and give overall normalization factors $\alpha_{B_{s}^{0} \rightarrow \mu^{+} \mu^{-}}=(8.6 \pm 1.1) \times 10^{-9}$ and $\alpha_{B_{d}^{0} \rightarrow \mu^{+} \mu^{-}}=(2.24 \pm 0.16) \times 10^{-9}$.

In the third step of the analysis each event is given a probability to be signal or background in a two-dimensional space. This probability is defined by two variables: the $\mu^{+} \mu^{-}$invariant mass and the so-called geometrical likelihood, GL. The GL is a mathematical combination of several variables mainly related to the geometry of the event. The definition of the GL variable is such that background events cluster around zero, and signal events will be uniformly distributed between zero and one. The optimization of the combination of variables that enters in the GL is performed using MC. However, the probability for a signal event to have a given value of GL is obtained from the data itself using $B_{q}^{0} \rightarrow h^{+} h^{-}$events. The probability for a background event to have a given GL is instead obtained by interpolating the events in the dimuon invariant mass sidebands. The results of the calibration of GL for signal and background are shown in figure 2.

The parameters needed to describe the invariant mass distribution for the signal events are extracted from the fit of the invariant mass distribution of $B_{q}^{0} \rightarrow h^{+} h^{-}$decays, and by interpolating the results obtained with dimuon resonances. The measured invariant mass resolution is $\sigma=(26.7 \pm 0.9) \mathrm{MeV} / c^{2}$.

\subsection{Results}

The observed distribution of GL vs $M\left(\mu^{+} \mu^{-}\right)$for the selected dimuon events is shown in figure 3. The sensitive region (i.e. the region from which most of the sensitivity to the $B_{s}^{0} \rightarrow \mu^{+} \mu^{-}$ and $B_{d}^{0} \rightarrow \mu^{+} \mu^{-}$branching fractions comes from) is defined by the 2-dimensional region GL $>0.5$ and $M_{B}-60 \mathrm{MeV} / \mathrm{c}^{2}<M\left(\mu^{+} \mu^{-}\right)<M_{B}+60 \mathrm{MeV} / \mathrm{c}^{2}$, with $M_{B}$ denoting either the $B_{s}^{0}$ or the $B_{d}^{0}$ mass. For the event counting, the invariant mass window given above is subdivided in six inter- 


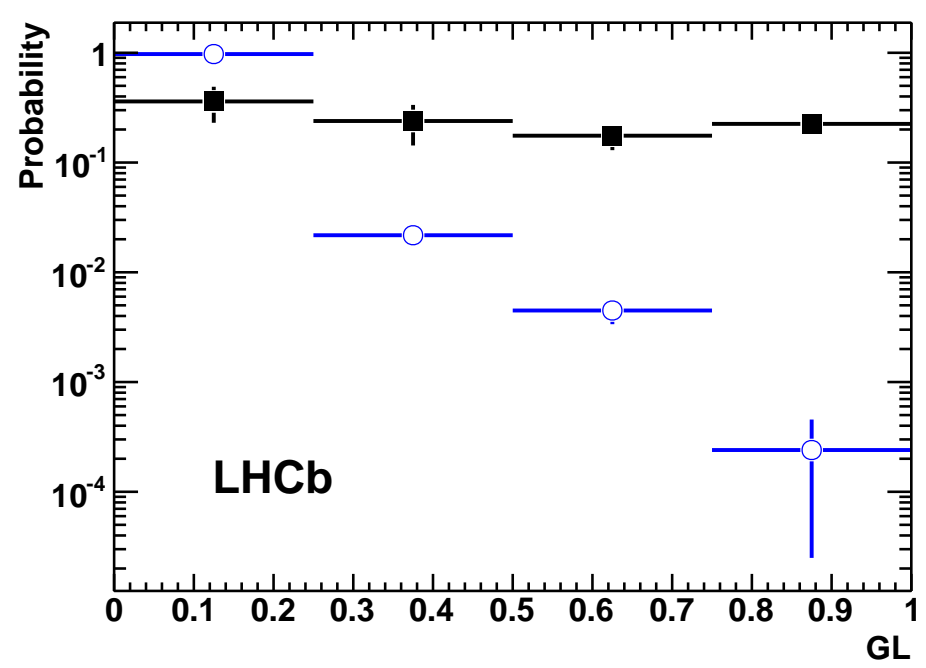

Figure 2: Probability of signal events in bins of GL obtained from the inclusive sample of $B_{q}^{0} \rightarrow h^{+} h^{-}$events (solid squares). The background probability (open circles) is obtained from the events in the sidebands of the $\mu \mu$ invariant mass distribution in the $B_{s}^{0}$ mass window. [7]

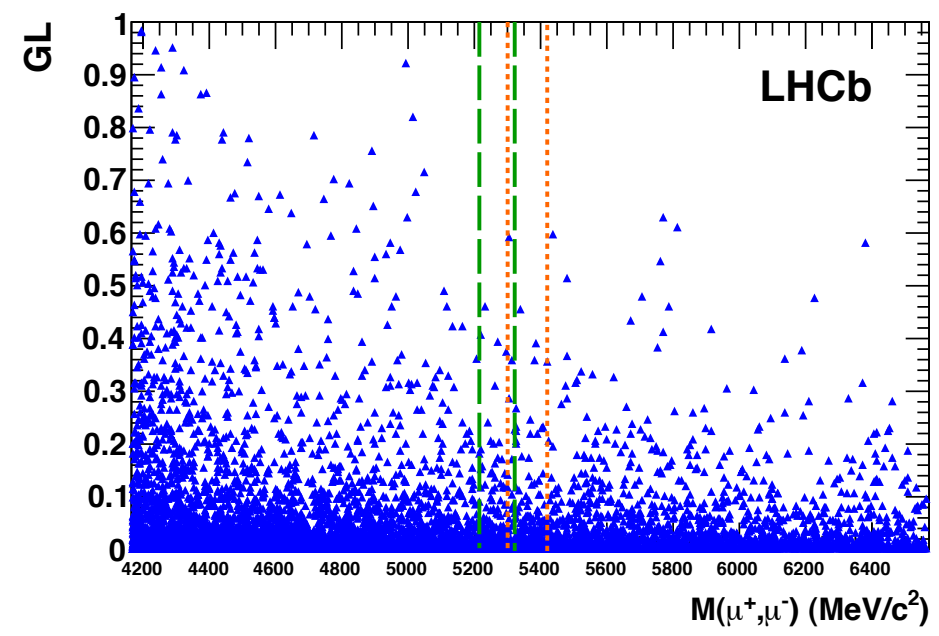

Figure 3: Observed distribution of selected dimuon events in the plane GL versus dimuon invariant mass.The green long-dashed (orange short-dashed) lines indicate the $\pm 60 \mathrm{MeV} / \mathrm{c}^{2}$ search window around the nominal $B_{d}\left(B_{s}\right)$ mass. [7]

vals, and the whole GL range is subdivided in four intervals. For each bin the number of observed event in data is computed, and the number of expected signal events, for a given BR hypothesis and luminosity, and the number of expected background events are evaluated. The amount of background is estimated by interpolation of the mass sidebands, in bins of GL. The compatibility of the observed distribution of events in all bins with the expected number for a given BR hypothesis is then computed using the modified frequentist approach method, also known as $\mathrm{CL}_{\mathrm{s}}$ method [6], which allows to exclude a given hypothesis at a given confidence level. The observed distribution 
of $\mathrm{CL}_{\mathrm{s}}$ versus $\mathrm{BR}$ can be seen in figure 4. The expected distributions of $C L_{\mathrm{s}}$ assuming the background only hypothesis are also shown in the same figure as a green area that covers the region of $\pm 1 \sigma$ of possible observations. The uncertainties in the signal and background likelihoods and normalization factors, including systematics, are propagated into the $\mathrm{CL}_{\mathrm{s}}$ evaluation.
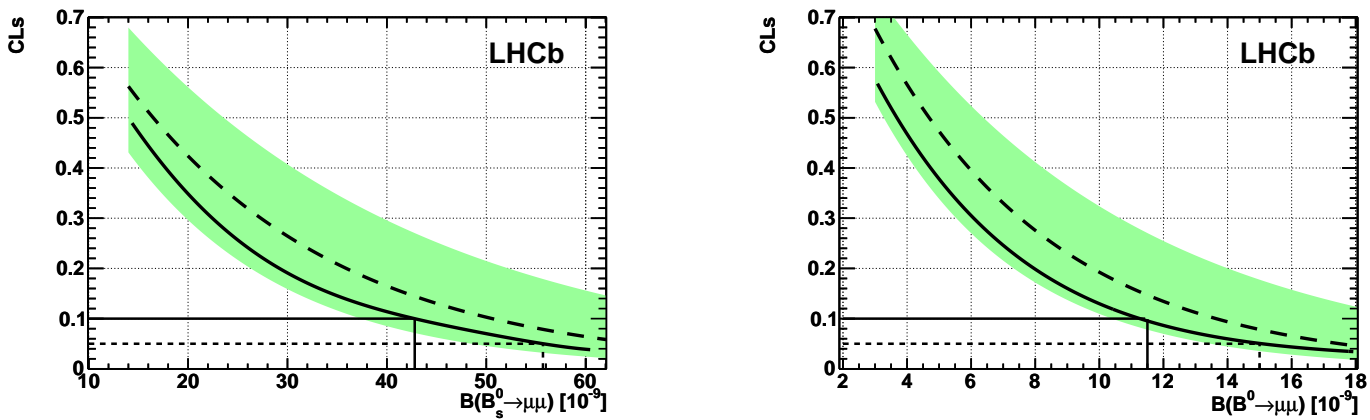

Figure 4: (a) Observed (solid curve) and expected (dashed curve) $\mathrm{CL}_{\mathrm{s}}$ values as a function of $B R\left(B_{S} \rightarrow\right.$ $\left.\mu^{+} \mu^{-}\right)$. The green band contains the $\pm 1 \sigma$ interval of possible results compatible with the expected value when only background is observed. The $90 \%$ (95\%) CL observed value is identified by the solid (dotted) line. (b) the same for $B R\left(B_{d}^{0} \rightarrow \mu^{+} \mu^{-}\right)$. [7]

Although not statistically significant, a slight deficit of events is observed in the most sensitive bins with respect to the background only hypothesis, which gives a small deviation between the observed and the expected $\mathrm{CL}_{\mathrm{s}}$. The upper limits are finally computed using the $\mathrm{CL}_{\mathrm{s}}$ distributions of figure 4. The results are [7]:

$$
\begin{aligned}
& \mathrm{BR}\left(B_{s}^{0} \rightarrow \mu^{+} \mu^{-}\right)<43(56) \times 10^{-9} \text { at } 90 \%(95 \%) \text { C.L. }, \\
& \operatorname{BR}\left(B_{d}^{0} \rightarrow \mu^{+} \mu^{-}\right)<12(15) \times 10^{-9} \text { at } 90 \%(95 \%) \text { C.L. },
\end{aligned}
$$

while the expected values for the upper limits are $\mathrm{BR}\left(B_{s}^{0} \rightarrow \mu^{+} \mu^{-}\right)<51(65) \times 10^{-9}$ and $\mathrm{BR}\left(B_{d}^{0} \rightarrow\right.$ $\left.\mu^{+} \mu^{-}\right)<14(18) \times 10^{-9}$ at $90 \%$ (95\%) C.L. The LHCb limits are similar to the best results from $\mathrm{CDF}$ [3] proving the effectiveness of the LHCb design and its excellent performances. With the ongoing LHC run, which is scheduled to last up to the end of year 2012, the LHCb experiment expects to collect up to $\sim 2 \mathrm{fb}^{-1}$. Assuming the present performance, this data set will allow for $B_{s}^{0} \rightarrow \mu^{+} \mu^{-}$to explore the full range down to the SM expectation.

\section{Study of $B_{d}^{0} \rightarrow K^{* 0} \mu^{+} \mu^{-}$decay}

The $B_{d}^{0} \rightarrow K^{* 0} \mu^{+} \mu^{-}$decay offers several observables which are very sensitive to physics phenomena beyond the SM [8]. One of the most powerful is the forward-backward asymmetry of $\mu^{+}$with respect to the $B_{d}^{0}$ direction in the dimuon rest frame, measured as a function of the squared dimuon invariant mass, $q^{2}$. The SM prediction for this quantity is determined by the interference of vector and axial-vector electroweak currents, and gives direct access to the phases of the Wilson coefficients $C_{7}, C_{9}$ and $C_{10}$. Particularly interesting is the region of low $q^{2}\left(<6 \mathrm{GeV}^{2} / c^{2}\right)$, where the uncertainties of hadronic form factors are under control. Current measurements [9], based on few hundreds of events, exhibit poor statistical accuracy and do not allow to exclude new physics scenarios (e.g flipped $C_{7}$ ). 
With the 2010 data sample, $\mathrm{LHCb}$ was able to obtain a clean mass peak with $23 \pm 6$ signal events, shown in figure 5. The observed rate, which is close to expectation [10], will allow us to perform with the first $\mathrm{fb}^{-1}$ of data the most precise asymmetry measurement. Systematic uncertainties result from a possible angular bias caused by a non-uniform detector acceptance and trigger and selection efficiencies. To measure these effects, the $B_{d}^{0} \rightarrow J / \psi\left(\mu^{+} \mu^{-}\right) K^{* 0}$ decay is the natural control channel, as it has the same final state as the signal.

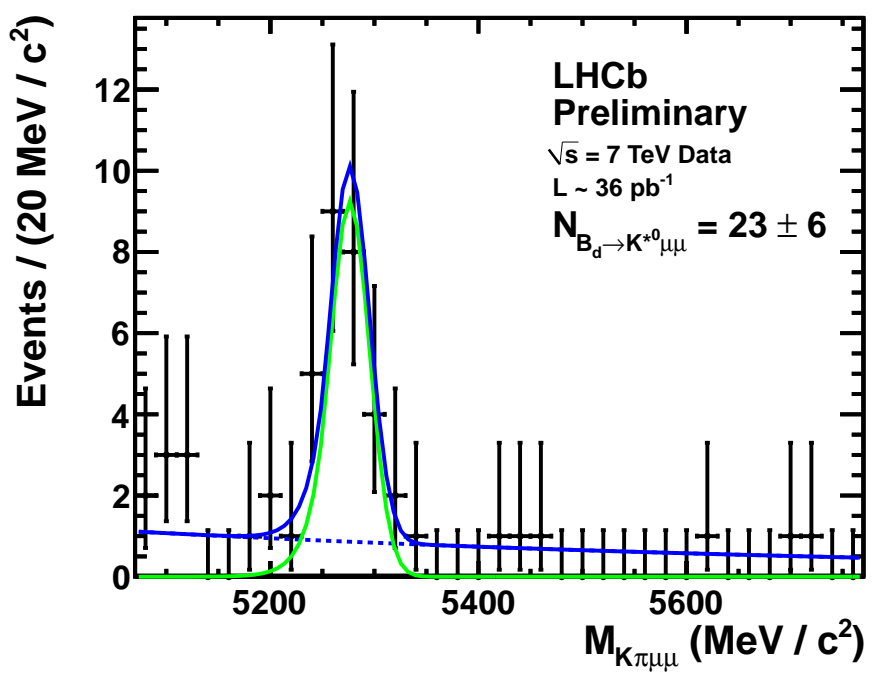

Figure 5: Observed $M_{K \pi \mu \mu}$ mass spectrum for events passing the $B_{d}^{0} \rightarrow K^{* 0} \mu^{+} \mu^{-}$selection.

\section{Summary and conclusions}

The study of FCNC decays of $B$ mesons, which can only happen through loop diagrams in the $\mathrm{SM}$, is a powerful handle to look for physics phenomena beyond the SM. With the data collected during year 2010, corresponding to an integrated luminosity of about $37 \mathrm{pb}^{-1}$, the LHCb experiment has demonstrated a very good potential in this field of research, due to its large acceptance and trigger efficiency, as well as the large $b \bar{b}$ cross-section in $p p$ collisions at $\sqrt{s}=7 \mathrm{TeV}$.

LHCb has searched for the rare decays $B_{s}^{0} \rightarrow \mu^{+} \mu^{-}$and $B_{d}^{0} \rightarrow \mu^{+} \mu^{-}$, and reached sensitivities similar to the existing limits from the Tevatron experiments. The observed events are compatible with the background expectations, and the upper limits are evaluated to be

$$
\begin{aligned}
& \operatorname{BR}\left(B_{s}^{0} \rightarrow \mu^{+} \mu^{-}\right)<56 \times 10^{-9} \text { at 95\% C.L. }, \\
& \operatorname{BR}\left(B_{d}^{0} \rightarrow \mu^{+} \mu^{-}\right)<15 \times 10^{-9} \text { at 95\% C.L.. }
\end{aligned}
$$

The LHC is expected to deliver a much larger sample of $p p$ collisions in 2011 and 2012. Given the low level of background in the most sensitive region of the parameter space, LHCb should be able to explore the interesting region of branching fractions down to the SM predictions.

LHCb has also shown a clean observation of the $B_{d}^{0} \rightarrow K^{* 0} \mu^{+} \mu^{-}$decay with 2010 data sample, which gives excellent prospects for measuring accurately the angular distribution of this decay with the first $\mathrm{fb}^{-1}$ of data, and thus probing the Lorentz structure of the FCNC processes. 


\section{References}

[1] S. R. Choudhury and N. Gaur, "Dileptonic decay of $B_{s}$ meson in SUSY models with large $\tan \beta$ " Phys. Lett. B 451, (1999) 86.

[2] G. Buchalla and A.J. Buras, "The rare decays $K \rightarrow \pi v \bar{v}, B \rightarrow X v \bar{v}$ and $B \rightarrow l^{+} l^{-}-$an update", Nucl. Phys. B 548 (1999) 309.

[3] T. Aaltonen et al. [CDF Collaboration], "Search for $B_{s} \rightarrow \mu^{+} \mu^{-}$and $B_{d}^{0} \rightarrow \mu^{+} \mu^{-}$decays in $3.7 \mathrm{fb}^{-1}$ of $p \bar{p}$ collisions with CDF II", CDF Public Note 9892.

[4] A.A. Alves et al. [LHCb Collaboration], “The LHCb detector at the LHC”, JINST 3 (2008) S08005, and references therein.

[5] R. Aaij et al. [LHCb Collaboration], "Measurement of $\sigma(p p \rightarrow b \bar{b} X)$ at $\sqrt{s}=7 \mathrm{TeV}$ in the forward region”, Phys. Lett. B 694 (2010) 209.

[6] A. Read, âĂIJPresentation of Search Results: The CLs TechniqueâĂİ, J. Phys. G 28 (2002) 2693.

[7] R. Aaij et al. [LHCb Collaboration], "Search for the decays $B_{s}^{0} \rightarrow \mu^{+} \mu^{-}$and $B_{d}^{0} \rightarrow \mu^{+} \mu^{-}$, Phys. Lett. B 699 (2011) 330.

[8] U. Egede et al., "New observables in the decay mode $\bar{B} \rightarrow \bar{K}^{* 0} l^{+} l^{-}$", JHEP 0811 (2008) 032.

[9] B. Aubert et al. [BABAR Collaboration], Phys. Rev. D 79 (2009) 031102; J.T. Wei et al. [BELLE Collaboration], Phys. Rev. Lett. 103 (2009) 171801; CDF Public note 10047.

[10] B. Adeva et al. [LHCb Collaboration], "Roadmap for selected key measurements of LHCb", arXiv:0912.4179v2. 